\title{
Fibonacci numbers which are products of two balancing numbers
}

\author{
Fatih Erduvan, Refik Keskin \\ Sakarya University, Mathematics Department, Sakarya, Turkey \\ erduvanmat@hotmail.com \\ rkeskin@sakarya.edu.tr \\ Submitted: September 11, 2018 \\ Accepted: June 9, 2019 \\ Published online: June 26, 2019
}

\begin{abstract}
The Fibonacci sequence $\left(F_{n}\right)$ is defined by $F_{0}=0, F_{1}=1$ and $F_{n}=$ $F_{n-1}+F_{n-2}$ for $n \geq 2$. The balancing number sequence $\left(B_{n}\right)$ is defined by $B_{0}=0, B_{1}=1$ and $B_{n}=6 B_{n-1}-B_{n-2}$ for $n \geq 2$. In this paper, we find all Fibonacci numbers which are products of two balancing numbers. Also we found all balancing numbers which are products of two Fibonacci numbers. More generally, taking $k, m, m$ as positive integers, it is proved that $F_{k}=B_{m} B_{n}$ implies that $(k, m, n)=(1,1,1),(2,1,1)$ and $B_{k}=F_{m} F_{n}$ implies that $(k, m, n)=(1,1,1),(1,1,2),(1,2,2),(2,3,4)$.
\end{abstract}

Keywords: Fibonacci number, balancing number, Diophantine equations, linear forms in logarithms.

$M S C: 11 \mathrm{~B} 39,11 \mathrm{~J} 86,11 \mathrm{D} 61$

\section{Introduction}

The Fibonacci sequence $\left(F_{n}\right)$ is defined as $F_{0}=0, F_{1}=1$ and $F_{n}=F_{n-1}+F_{n-2}$ for $n \geq 2 . F_{n}$ is called the $n$-th Fibonacci number. It well known that

$$
F_{n}=\frac{\alpha^{n}-\beta^{n}}{\sqrt{5}}
$$


for every $n \geq 0$, where $\alpha=\frac{1+\sqrt{5}}{2}$ and $\beta=\frac{1-\sqrt{5}}{2}$, which are the roots of the characteristic equations $x^{2}-x-1=0$. It is well known that

$$
\alpha^{n-2} \leq F_{n} \leq \alpha^{n-1}
$$

for all $n \geq 1$. The inequality (1.1) can be proved by induction. It can be seen that $1<\alpha<2$ and $-1<\beta<0$. For more information about the Fibonacci sequence and its applications, one can see [7]. A positive integer $n$ is called a balancing number if the equation

$$
1+2+\cdots+(n-1)=(n+1)+\cdots+(n+r)
$$

holds for some positive integer $r$. The sequence of balancing numbers $\left(B_{n}\right)$ satisfys recurrence relation $B_{n}=6 B_{n-1}-B_{n-2}$ for $n \geq 2$ with initial conditions $B_{0}=$ $0, B_{1}=1 . B_{n}$ is called the $n$-th balancing number. We have the Binet formula

$$
B_{n}=\frac{\lambda^{n}-\delta^{n}}{4 \sqrt{2}}
$$

where $\lambda=3+2 \sqrt{2}$ and $\delta=3-2 \sqrt{2}$, which are the roots of the characteristic equations $x^{2}-6 x+1=0$. Therefore,

$$
B_{n}<\frac{\lambda^{n}}{4 \sqrt{2}} .
$$

It can be seen that $5<\lambda<6,0<\delta<1$ and $\lambda \delta=1$. Moreover, it holds that

$$
\lambda^{n-1} \leq B_{n}<\lambda^{n}
$$

for all $n \geq 1$. This inequality can be proved by noting the facts that $\lambda^{n}=\lambda B_{n}-$ $B_{n-1}$ and $B_{n}-\lambda^{n-1}=B_{n}-\left(\lambda B_{n-1}-B_{n-2}\right)=6 B_{n-1}-B_{n-2}-\left(\lambda B_{n-1}-B_{n-2}\right)=$ $(6-\lambda) B_{n-1}>0$ for all $n \geq 2$. Clearly, the identity (1.3) holds for $n=1$. For more information about the sequence of balancing numbers, see $[6,9,10]$. A different definition is given by Szakács [12]. A positive integer $n$ is called a multiplying balancing number if the equation

$$
1 \cdot 2 \cdots(n-1)=(n+1)(n+2) \cdots(n+r)
$$

holds for some positive integer $r$. The number $r$ is called the balancer corresponding to multiplying balancing number $n$. In [12], it is shown that the only multiplying balancing number is $n=7$ with the balancer $r=3$. For some other generalization of balancing numbers, the interested readers can consult [11] and the references there. In [3], the authors have found all Fibonacci numbers or Pell numbers which are products of two numbers from the other sequence. Taking $k, m$ and $n$ are positive integer, they showed that $F_{k}=P_{m} P_{n}$ implies that $k=1,2,3,5,12$ and $P_{k}=F_{m} F_{n}$ implies that $k=1,2,3,7$, where $\left(P_{n}\right)$ is the Pell sequence defined by $P_{0}=0, P_{1}=1$ and $P_{n}=2 P_{n-1}+P_{n-2}$ for $n \geq 2$. In this study, we determine all solutions of the equation

$$
F_{k}=B_{m} B_{n}
$$


and

$$
B_{k}=F_{m} F_{n}
$$

in positive integers $k, n, m$. More generally, taking $k, m, m$ as positive integers, it is proved that $F_{k}=B_{m} B_{n}$ implies that $(k, m, n)=(1,1,1),(2,1,1)$ and $B_{k}=F_{m} F_{n}$ implies that $(k, m, n)=(1,1,1),(1,1,2),(1,2,2),(2,3,4)$.

Our study can be viewed as a continuation of the previous work on this subject. We follow the approach and the method presented in [3]. In Section 2, we introduce necessary lemmas and theorems. Then in Section 3, we prove our main theorem.

\section{Auxiliary results}

In [3], in order to solve Diophantine equations of the form (1.4) and (1.5), the authors have used Baker's theory of lower bounds for a nonzero linear form in logarithms of algebraic numbers. Since such bounds are of crucial importance in effectively solving of Diophantine equations of the similar form, we start with recalling some basic notions from algebraic number theory.

Let $\eta$ be an algebraic number of degree $d$ with minimal polynomial

$$
a_{0} x^{d}+a_{1} x^{d-1}+\cdots+a_{d}=a_{0} \prod_{i=1}^{d}\left(X-\eta^{(i)}\right) \in \mathbb{Z}[x],
$$

where the $a_{i}$ 's are relatively prime integers with $a_{0}>0$ and $\eta^{(i)}$ 's are conjugates of $\eta$. Then

$$
h(\eta)=\frac{1}{d}\left(\log a_{0}+\sum_{i=1}^{d} \log \left(\max \left\{\left|\eta^{(i)}\right|, 1\right\}\right)\right)
$$

is called the logarithmic height of $\eta$. In particularly, if $\eta=a / b$ is a rational number with $\operatorname{gcd}(a, b)=1$ and $b>1$, then $h(\eta)=\log (\max \{|a|, b\})$.

The following properties of logarithmic height are found in many works stated in the references:

$$
\begin{aligned}
h(\eta \pm \gamma) & \leq h(\eta)+h(\gamma)+\log 2, \\
h\left(\eta \gamma^{ \pm 1}\right) & \leq h(\eta)+h(\gamma), \\
h\left(\eta^{s}\right) & =|s| h(\eta) .
\end{aligned}
$$

The following theorem is deduced from Corollary 2.3 of Matveev [8] and provides a large upper bound for the subscript $n$ in the equations (1.4) and (1.5)(also see Theorem 9.4 in [2]).

Theorem 2.1. Assume that $\gamma_{1}, \gamma_{2}, \ldots, \gamma_{t}$ are positive real algebraic numbers in a real algebraic number field $\mathbb{K}$ of degree $D, b_{1}, b_{2}, \ldots, b_{t}$ are rational integers, and

$$
\Lambda:=\gamma_{1}^{b_{1}} \ldots \gamma_{t}^{b_{t}}-1
$$


is not zero. Then

$$
|\Lambda|>\exp \left(-1.4 \cdot 30^{t+3} \cdot t^{4.5} \cdot D^{2}(1+\log D)(1+\log B) A_{1} A_{2} \ldots A_{t}\right),
$$

where

$$
B \geq \max \left\{\left|b_{1}\right|, \ldots,\left|b_{t}\right|\right\},
$$

and $A_{i} \geq \max \left\{D h\left(\gamma_{i}\right),\left|\log \gamma_{i}\right|, 0.16\right\}$ for all $i=1, \ldots, t$.

The following lemma was proved by Dujella and Pethô [5] and is a variation of a lemma of Baker and Davenport [1]. This lemma will be used to reduce the upper bound for the subscript $n$ in the equations (1.4) and (1.5). In the following lemma, the function $\|\cdot\|$ denotes the distance from $x$ to the nearest integer. That is, $\|x\|=\min \{|x-n|: n \in \mathbb{Z}\}$ for any real number $x$.

Lemma 2.2. Let $M$ be a positive integer, let $p / q$ be a convergent of the continued fraction of the irrational number $\gamma$ such that $q>6 M$, and let $A, B$, $\mu$ be some real numbers with $A>0$ and $B>1$. Let $\epsilon:=\|\mu q\|-M\|\gamma q\|$. If $\epsilon>0$, then there exists no solution to the inequality

$$
0<|u \gamma-v+\mu|<A B^{-w}
$$

in positive integers $u, v$, and $w$ with

$$
u \leq M \quad \text { and } \quad w \geq \frac{\log (A q / \epsilon)}{\log B} .
$$

The following theorems are given in [2] and [4], respectively.

Theorem 2.3. The only perfect powers in the Fibonacci sequence are $F_{0}=0, F_{1}=$ $F_{2}=1, F_{6}=8$ and $F_{12}=144$.

Theorem 2.4. For any given positive integers $y$ and $l \geq 2$, the equation $B_{m}=y^{l}$ has no solution for integers $m \geq 2$.

\section{Main theorems}

Theorem 3.1. The Diophantine equation $F_{k}=B_{m} B_{n}$ has only the solutions

$$
(k, m, n)=(1,1,1),(2,1,1)
$$

in positive integers.

Proof. Assume that the equation $F_{k}=B_{m} B_{n}$ holds. If $m=n$, we have $F_{k}=B_{n}^{2}$, which is possible only for $k=1,2$, and $n=1$ by Theorem 2.3. In this case, $(k, m, n)=(1,1,1),(2,1,1)$. Therefore, we assume that $1 \leq m<n$. Let $n \leq 30$. Then, by using the Mathematica program, we see that $k \leq 214$. In that case, with the help of Mathematica program, we obtain only the solutions $(k, m, n)=$ 
$(1,1,1),(2,1,1)$ in the range $1 \leq m<n \leq 30$. This takes a little time. From now on, assume that $n>30$. Using the inequality (1.1) and (1.2), we get the inequality

$$
\alpha^{k-2} \leq F_{k}=B_{m} B_{n}<\lambda^{n+m} / 32 .
$$

From this, it follows that

$$
\alpha^{k}=\alpha^{2} \alpha^{k-2}<32 \alpha^{k-2}<\lambda^{n+m}<\left(\alpha^{4}\right)^{n+m},
$$

which yields to $k<4(n+m)<8 n$. On the other hand, $\lambda^{m+n-2} \leq B_{m} B_{n}=F_{k} \leq$ $\alpha^{k-1}<\lambda^{k-1}$ by (1.1) and (1.3). From this, we get $m+n-1<k$, which implies that $k>n$.

Since

$$
\frac{\alpha^{k}-\beta^{k}}{\sqrt{5}}=F_{k}=B_{m} B_{n}=\frac{\lambda^{n+m}+\delta^{n+m}-\lambda^{n} \delta^{m}-\lambda^{m} \delta^{n}}{32},
$$

we get

$$
\frac{\beta^{k}}{\sqrt{5}}-\frac{\lambda^{n} \delta^{m}+\lambda^{m} \delta^{n}-\delta^{n+m}}{32}=\frac{\alpha^{k}}{\sqrt{5}}-\frac{\lambda^{n+m}}{32} .
$$

Taking absolute values, we obtain

$$
\begin{aligned}
\left|\frac{\alpha^{k}}{\sqrt{5}}-\frac{\lambda^{n+m}}{32}\right| & =\left|\frac{\beta^{k}}{\sqrt{5}}-\frac{\lambda^{n} \delta^{m}+\lambda^{m} \delta^{n}-\delta^{n+m}}{32}\right| \leq \frac{|\beta|^{k}}{\sqrt{5}}+\frac{\lambda^{n} \delta^{m}+\lambda^{m} \delta^{n}+\delta^{n+m}}{32} \\
& =\frac{32|\beta|^{k}+\sqrt{5}\left(\lambda^{n-m}+\delta^{n-m}+\delta^{n+m}\right)}{32 \sqrt{5}} \\
& <\frac{\sqrt{5}+\sqrt{5}\left(\lambda^{n-m}+2\right)}{32 \sqrt{5}}<\frac{\sqrt{5}+2 \sqrt{5} \lambda^{n-m}}{32 \sqrt{5}} \\
& <\frac{1+2 \lambda^{n-m}}{32}<\frac{\lambda^{n-m+1}}{32},
\end{aligned}
$$

where we have used the fact that $0<\delta<1, \lambda>2, \lambda \delta=1$, and $32|\beta|^{k}<\sqrt{5}$ for $k>n>30$. If we divide both sides of the above inequality by $\frac{\lambda^{n+m}}{32}$, we get

$$
\left|\frac{32}{\sqrt{5}} \alpha^{k} \lambda^{-(n+m)}-1\right|<\frac{1}{\lambda^{2 m-1}}
$$

Now, let us apply Theorem 2.1 with $\gamma_{1}:=32 / \sqrt{5}, \gamma_{2}:=\alpha, \gamma_{3}:=\lambda$ and $b_{1}:=$ $1, b_{2}:=k, \quad b_{3}:=-(n+m)$. Note that the numbers $\gamma_{1}, \gamma_{2}$, and $\gamma_{3}$ are positive real numbers and elements of the field $\mathbb{K}=\mathbb{Q}(\sqrt{2}, \sqrt{5})$. It is obvious that the degree of the field $\mathbb{K}$ is 4 . So $D=4$. Now, we show that $\Lambda_{1}:=\frac{32}{\sqrt{5}} \alpha^{k} \lambda^{-(n+m)}-1$ is nonzero. For, if $\Lambda_{1}=0$, then we get

$$
\alpha^{k} \lambda^{-(n+m)}=\alpha^{k} \delta^{n+m}=\sqrt{5} / 32 .
$$


It is seen that $\sqrt{5} / 32$ is not a algebraic integer although $\alpha^{k} \delta^{n+m}$ is an algebraic integer. This is a contradiction. Moreover, since

$$
\begin{aligned}
& h\left(\gamma_{1}\right)=h(32 / \sqrt{5})=\frac{1}{2}(\log 5+2 \log (32 / \sqrt{5}))=3.4657 \ldots, \\
& h\left(\gamma_{2}\right)=\frac{\log \alpha}{2}=\frac{0.4812 \ldots}{2}
\end{aligned}
$$

and

$$
h\left(\gamma_{3}\right)=\frac{\log \lambda}{2}=\frac{1.76275 \ldots}{2}
$$

by (2.1), we can take $A_{1}:=14, A_{2}:=1$ and $A_{3}=3.6$. Also, since $k<8 n$, we can take $B:=\max \{1,|k|,|-(n+m)|\}=8 n$. Thus, taking into account the inequality (3.1) and using Theorem 2.1, we obtain

$$
\frac{1}{\lambda^{2 m-1}}>\left|\Lambda_{1}\right|>\exp \left(-1.4 \cdot 30^{6} \cdot 3^{4.5} \cdot 4^{2}(1+\log 4)(1+\log 8 n)(14)(3.6)\right),
$$

and so

$$
(2 m-1) \log \lambda<1.4 \cdot 30^{6} \cdot 3^{4.5} \cdot 4^{2}(1+\log 4)(1+\log 8 n)(14)(3.6) .
$$

By a simple computation, it follows that

$$
2 m \log \lambda<2.7554 \cdot 10^{14}(1+\log 8 n)+\log \lambda .
$$

Now, we apply Theorem 2.1 a second time. Rearranging the equation $F_{k}=B_{n} B_{m}$ as

$$
\frac{\beta^{k}}{\sqrt{5} B_{m}}-\frac{\delta^{n}}{4 \sqrt{2}}=\frac{\alpha^{k}}{\sqrt{5} B_{m}}-\frac{\lambda^{n}}{4 \sqrt{2}},
$$

and taking absolute values, we obtain

$$
\left|\frac{\alpha^{k}}{\sqrt{5} B_{m}}-\frac{\lambda^{n}}{4 \sqrt{2}}\right|=\left|\frac{\beta^{k}}{\sqrt{5} B_{m}}-\frac{\delta^{n}}{4 \sqrt{2}}\right| \leq \frac{|\beta|^{k}}{\sqrt{5} B_{m}}+\frac{\delta^{n}}{4 \sqrt{2}}<\frac{1}{\sqrt{5} B_{m}}+\frac{1}{4 \sqrt{2}}<1,
$$

where we used the fact that $|\beta|<1$ and $0<\delta<1$. Dividing both sides of the above inequality by $\lambda^{n} / 4 \sqrt{2}$, we get

$$
\left|\frac{4 \sqrt{2} \alpha^{k} \lambda^{-n}}{\sqrt{5} B_{m}}-1\right|<\frac{4 \sqrt{2}}{\lambda^{n}}<\frac{6}{\lambda^{n}} .
$$

Taking $\gamma_{1}:=\alpha, \gamma_{2}:=\lambda, \gamma_{3}:=\sqrt{5} B_{m} / 4 \sqrt{2}$, and $b_{1}:=k, b_{2}:=-n, b_{3}:=-1$, we can apply Theorem 2.1. The numbers $\gamma_{1}, \gamma_{2}$, and $\gamma_{3}$ are positive real numbers and elements of the field $\mathbb{K}=\mathbb{Q}(\sqrt{2}, \sqrt{5})$ and so $D=4$. In a similar manner, one can verify that $\Lambda_{2}=4 \sqrt{2} \alpha^{k} \lambda^{-n} / B_{m}-1 \neq 0$. Also, since $h\left(\gamma_{1}\right)=\frac{\log \alpha}{2}=\frac{0.4812 \ldots}{2}$ and 
$h\left(\gamma_{2}\right)=\frac{\log \lambda}{2}=\frac{1.76275 \ldots}{2}$ by $(2.1)$, we can take $A_{1}:=1$ and $A_{2}=3.6$. The number $\sqrt{5} B_{m} / 4 \sqrt{2}$ is a root of the polynomial $32 X^{2}-5 B_{m}^{2}$. Thus, using the properties $(2.2),(2.3)$ and $(2.4)$, it is seen that

$$
\begin{aligned}
h\left(\gamma_{3}\right) & \leq \frac{1}{2}\left(\log 32+2 \log \left(\frac{\sqrt{5} B_{m}}{4 \sqrt{2}}\right)\right)=\log \left(\sqrt{5} B_{m}\right) \leq \log \left(\sqrt{5} \lambda^{m} / 4 \sqrt{2}\right) \\
& <m \log \lambda
\end{aligned}
$$

by (1.2). So we can take $A_{3}:=4 m \log \lambda$. Since $k<8 n$, it follows that $B:=8 n>$ $\max \{|k|,|-n|,|-1|\}$. Thus, taking into account the inequality (3.3) and using Theorem 2.1, we obtain

$$
\frac{6}{\lambda^{n}}>\left|\Lambda_{2}\right|>\exp ((-C)(1+\log 4)(1+\log 8 n)(3.6) 4 m \log \lambda),
$$

or

$$
n \log \lambda-\log 6<C(1+\log 4)(1+\log 8 n)(3.6) 4 m \log \lambda,
$$

where $C=1.4 \cdot 30^{6} \cdot 3^{4.5} \cdot 4^{2}$. Inserting the inequality (3.2) into the last inequality, a computer search with Mathematica gives us that $n<3.52 \cdot 10^{31}$.

Now, let us try to reduce the upper bound on $n$ by applying Lemma 2.2. Let

$$
z_{1}:=k \log \alpha-(n+m) \log \lambda+\log (32 / \sqrt{5}) .
$$

Then

$$
\left|1-e^{z_{1}}\right|<\frac{1}{\lambda^{2 m-1}}
$$

by (3.1). If $z_{1}>0$, then we have the inequality

$$
\left|z_{1}\right|=z_{1}<e^{z_{1}}-1=\left|1-e^{z_{1}}\right|<\frac{1}{\lambda^{2 m-1}}
$$

since $x<e^{x}-1$ for $x>0$. If $z_{1}<0$, then

$$
1-e^{z_{1}}=\left|1-e^{z_{1}}\right|<\frac{1}{\lambda^{2 m-1}}<\frac{1}{2} .
$$

From this, we get $e^{z_{1}}>\frac{1}{2}$ and therefore

$$
e^{\left|z_{1}\right|}=e^{-z_{1}}<2
$$

Consequently, we get

$$
\left|z_{1}\right|<e^{\left|z_{1}\right|}-1=e^{\left|z_{1}\right|}\left|1-e^{z_{1}}\right|<\frac{2}{\lambda^{2 m-1}} .
$$

In both cases, the inequality

$$
\left|z_{1}\right|<\frac{2}{\lambda^{2 m-1}}
$$


holds. That is,

$$
0<|k \log \alpha-(n+m) \log \lambda+\log (32 / \sqrt{5})|<\frac{2}{\lambda^{2 m-1}} .
$$

Dividing this inequality by $\log \lambda$, we get

$$
0<\left|k\left(\frac{\log \alpha}{\log \lambda}\right)-(n+m)+\left(\frac{\log (32 / \sqrt{5})}{\log \lambda}\right)\right|<6.62 \cdot \lambda^{-2 m} .
$$

Take $\gamma:=\frac{\log \alpha}{\log \lambda} \notin \mathbb{Q}$ and $M:=2.82 \cdot 10^{32}$. Then we found that $q_{63}$, the denominator of the $63^{\text {th }}$ convergent of $\gamma$ exceeds $6 M$. Moreover,

$$
u:=k<8 n<8 \cdot 3.52 \cdot 10^{31}<M \text {. }
$$

Now take

$$
\mu:=\frac{\log (32 / \sqrt{5})}{\log \lambda}
$$

In this case, a quick computation with Mathematica gives us the inequality

$$
0<\epsilon=\left\|\mu q_{63}\right\|-M\left\|\gamma q_{63}\right\| \leq 0.408068 \text {. }
$$

Let $A:=6.62, B:=\lambda$ and $w:=2 m$ in Lemma 2.2. Thus, with the help of Mathematica, we can say that the inequality (3.5) has no solution for

$$
2 m=w \geq \frac{\log \left(A q_{63} / \epsilon\right)}{\log B} \geq 45.04933 .
$$

So

$$
m \leq 22 \text {. }
$$

Substituting this upper bound for $m$ into (3.4), we obtain $n<7.255727 \cdot 10^{16}$.

Now, let

$$
z_{2}:=k \log \alpha-n \log \lambda+\log \left(\frac{4 \sqrt{2}}{\sqrt{5} B_{m}}\right) .
$$

In this case, taking into account that $n>30$, it is seen that

$$
\left|1-e^{z_{2}}\right|<\frac{6}{\lambda^{n}}<\frac{1}{4}
$$

by $(3.3)$. If $z_{2}>0$, then

$$
\left|z_{2}\right|=z_{2}<e^{z_{2}}-1=\left|e^{z_{2}}-1\right|<\frac{6}{\lambda^{n}} .
$$

If $z_{2}<0$, then $1-e^{z_{2}}=\left|1-e^{z_{2}}\right|<\frac{1}{4}$. Therefore, we get $e^{z_{2}}>\frac{3}{4}$ and so $e^{\left|z_{2}\right|}=e^{-z_{2}}<\frac{4}{3}$. By using (3.7), we get

$$
0<\left|z_{2}\right|<e^{\left|z_{2}\right|}-1=e^{\left|z_{2}\right|}\left|1-e^{z_{2}}\right|<\frac{4}{3} \cdot \frac{6}{\lambda^{n}}=\frac{8}{\lambda^{n}} .
$$


Therefore, it holds that

$$
\left|z_{2}\right|<\frac{8}{\lambda^{n}}
$$

That is,

$$
0<\left|k \log \alpha-n \log \lambda+\log \left(\frac{4 \sqrt{2}}{\sqrt{5} B_{m}}\right)\right|<\frac{8}{\lambda^{n}} .
$$

Dividing both sides of the above inequality by $\log \lambda$, we get

$$
0<\left|k\left(\frac{\log \alpha}{\log \lambda}\right)-n+\frac{\log \left(\frac{4 \sqrt{2}}{\sqrt{5} B_{m}}\right)}{\log \lambda}\right|<4.54 \cdot \lambda^{-n} .
$$

Putting $\gamma:=\frac{\log \alpha}{\log \lambda}$ and taking $M:=5.81 \cdot 10^{17}$, we found that $q_{39}$, the denominator of the $39^{\text {th }}$ convergent of $\gamma$ exceeds $6 M$. Note that $u:=k<8 n<8 \cdot 7.25727 \cdot 10^{16}<$ M. Taking

$$
\mu:=\frac{\log \left(\frac{4 \sqrt{2}}{\sqrt{5} B_{m}}\right)}{\log \lambda}
$$

and considering the fact that $m \leq 22$ by (3.6), a quick computation with Mathematica gives us the inequality

$$
0<\epsilon=\left\|\mu q_{39}\right\|-M\left\|\gamma q_{39}\right\| \leq 0.467267
$$

for all $m \in[1,22]$. Let $A:=4.54, B:=\lambda$ and $w:=n$ in Lemma 2.2. Thus, with the help of Mathematica, we can say that the inequality (3.8) has no solution for

$$
n=w \geq \frac{\log \left(A q_{39} / \epsilon\right)}{B} \geq \frac{\log \left(A q_{39} / 0.467267\right)}{B} \geq 25.6246 .
$$

Therefore $n \leq 25$. This contradicts our assumption that $n>30$. Thus, the proof is completed.

Theorem 3.2. The Diophantine equation $B_{k}=F_{m} F_{n}$ has only the solutions $(k, m, n)=(1,1,1),(1,1,2),(1,2,2),(2,3,4)$ in positive integers.

Proof. Assume that $B_{k}=F_{m} F_{n}$ for some positive integers $k, m, n$. Let $n=m$. Then $B_{k}=F_{m}^{2}$. Therefore $k=1$ by Theorem 2.4. So we get $(k, m, n)=$ $(1,1,1),(1,2,2)$. Now assume that $1 \leq m<n \leq 107$. Then $k \leq 58$ and we get the solutions $(k, m, n)=(1,1,1),(1,1,2),(1,2,2),(2,3,4)$ by using Mathematica. So assume that $n>107$. Then $k \geq 59$. Since

$$
\left(\alpha^{3}\right)^{k-1}<\lambda^{k-1}<B_{k}=F_{m} F_{n} \leq \alpha^{n+m-2}
$$

by (1.1) and (1.3), it follows that $3(k-1)<n+m-2<2(n-1)$, which implies that $k<n$. In a similar manner, we see that $k>(m+n) / 4>108 / 4=27$. Since $B_{k}=F_{n} F_{m}$, we get

$$
\frac{\lambda^{k}}{4 \sqrt{2}}-\frac{\alpha^{m+n}}{5}=\frac{\delta^{k}}{4 \sqrt{2}}-\frac{\alpha^{n} \beta^{m}+\alpha^{m} \beta^{n}-\beta^{n+m}}{5}
$$




$$
=\frac{5 \delta^{k}+4 \sqrt{2}\left(\alpha^{n} \beta^{m}+\alpha^{m} \beta^{n}-\beta^{n+m}\right)}{20 \sqrt{2}} .
$$

Taking absolute values, it is seen that

$$
\begin{aligned}
\left|\frac{\lambda^{k}}{4 \sqrt{2}}-\frac{\alpha^{m+n}}{5}\right| & \leq \frac{5 \delta^{k}+4 \sqrt{2}\left(\alpha^{n}|\beta|^{m}+\alpha^{m}|\beta|^{n}+|\beta|^{m}\right)}{20 \sqrt{2}} \\
& =\frac{4 \sqrt{2} \alpha^{n-m}+5 \delta^{k}+4 \sqrt{2}\left(|\beta|^{n-m}+|\beta|^{n+m}\right)}{20 \sqrt{2}} \\
& \leq \frac{4 \sqrt{2} \alpha^{n-m}+4 \sqrt{2}}{20 \sqrt{2}} \\
& <\frac{4 \sqrt{2}\left(\alpha^{n-m}+1\right)}{20 \sqrt{2}}<\frac{\alpha^{n-m}+1}{5}<\frac{\alpha^{n-m+1}}{5},
\end{aligned}
$$

where we use the fact that $5 \delta^{k}+4 \sqrt{2}\left(|\beta|^{n-m}+|\beta|^{n+m}\right) \leq 4 \sqrt{2}$ for $k>27$ and $n>107$. Dividing both side of this inequality by $\alpha^{n+m} / 5$, we get

$$
\left|\frac{5 \lambda^{k} \alpha^{-(n+m)}}{4 \sqrt{2}}-1\right|<\frac{1}{\alpha^{2 m-1}} .
$$

Now we apply Matheev's theorem. Let $\gamma_{1}:=\frac{5}{4 \sqrt{2}}, \gamma_{2}:=\lambda, \gamma_{3}:=\alpha, b_{1}:=1, b_{2}:=$ $k, b_{3}:=-(n+m)$. The numbers $\gamma_{1}, \gamma_{2}, \gamma_{3}$ are real numbers and elements of the field $\mathbb{K}=\mathbb{Q}(\sqrt{2}, \sqrt{5})$. So $D=4$. Now we show that $\Lambda_{3}=\left(5 \lambda^{k} \alpha^{-(n+m)}\right) / 4 \sqrt{2}-1$ is nonzero. For, if $\Lambda_{3}=0$, then $\lambda^{k} \alpha^{-(m+n)}=4 \sqrt{2} / 5$. But this is impossible since $4 \sqrt{2} / 5$ is not an algebraic integer although $\lambda^{k} \alpha^{-(m+n)}$ is an algebraic integer. It can be seen that

$$
h\left(\gamma_{1}\right)=h(5 / 4 \sqrt{2})=\frac{1}{2}(\log 32)=1.7328 \ldots,
$$

$h\left(\gamma_{2}\right)=h(\lambda)=(1.76275) / 2$ and $h\left(\gamma_{3}\right)=h(\alpha)=(0.4812) / 2$. Therefore we can take $A_{1}:=7, A_{2}:=3.6, A_{3}:=1$ and $B:=2 n \geq \max \{1,|k|,|-(n+m)|\}$. Thus, taking into account the inequality (3.9) and using Theorem 2.1, we obtain

$$
\frac{1}{\alpha^{2 m-1}}>\left|\Lambda_{3}\right|>\exp \left(\left(-1.4 \cdot 30^{6} \cdot 3^{4.5} \cdot 4^{2}(1+\log 4)(1+\log 2 n) \cdot 7 \cdot 3.6 \cdot 1\right),\right.
$$

and so

$$
(2 m-1) \log \alpha<\left(1.37767 \cdot 10^{14}\right) \cdot(1+\log 2 n) .
$$

Then it follows that

$$
2 m \log \alpha<\left(1.37767 \cdot 10^{14}\right)(1+\log 2 n)+\log \alpha .
$$

Now, writing the equation $B_{k}=F_{m} F_{n}$ as

$$
\frac{\lambda^{k}}{4 \sqrt{2} F_{m}}-\frac{\alpha^{n}}{\sqrt{5}}=\frac{\delta^{k}}{4 \sqrt{2} F_{m}}-\frac{\beta^{n}}{\sqrt{5}},
$$


and taking absolute values, we get

$$
\left|\frac{\lambda^{k}}{4 \sqrt{2} F_{m}}-\frac{\alpha^{n}}{\sqrt{5}}\right| \leq \frac{\delta^{k}}{4 \sqrt{2} F_{m}}+\frac{|\beta|^{n}}{\sqrt{5}}<1 .
$$

By dividing both side of this inequality by $\alpha^{n} / \sqrt{5}$, we obtain

$$
\left|\frac{\lambda^{k} \sqrt{5} \alpha^{-n}}{4 \sqrt{2} F_{m}}-1\right|<\frac{\sqrt{5}}{\alpha^{n}}<\frac{3}{\alpha^{n}} .
$$

Take $\gamma_{1}:=\lambda, \gamma_{2}:=\alpha, \gamma_{3}:=\left(4 \sqrt{2} F_{m}\right) / \sqrt{5}, b_{1}:=k, b_{2}:=-n, b_{3}:=-1$. Clearly, the numbers $\gamma_{1}, \gamma_{2}, \gamma_{3}$ are real numbers and elements of the field $\mathbb{K}=\mathbb{Q}(\sqrt{2}, \sqrt{5})$ and so $D=4$. It can be seen that

$$
\Lambda_{4}=\frac{\lambda^{k} \sqrt{5} \alpha^{-n}}{4 \sqrt{2} F_{m}}-1
$$

is nonzero. On the other hand, $h\left(\gamma_{1}\right)=h(\lambda)=(1.76275 \ldots) / 2$ and $h\left(\gamma_{2}\right)=$ $h(\alpha)=(0.4882 \ldots) / 2$. Since $\left(4 \sqrt{2} F_{m}\right) / \sqrt{5}$ is a root of the polynomial $5 x^{2}-32 F_{m}^{2}$, it follows that

$$
\begin{aligned}
h\left(\gamma_{3}\right) & \leq \frac{1}{2}\left(\log 5+2 \log \left(4 \sqrt{2} F_{m} / \sqrt{5}\right)\right)=\log \left(4 \sqrt{2} F_{m}\right)=\log (4 \sqrt{2})+\log F_{m} \\
& <1.74+(m-1) \log \alpha<1.26+m \log \alpha,
\end{aligned}
$$

and so we can take $A_{3}:=4(1.26+m \log \alpha)$. Let $A_{1}:=3.6, A_{2}:=1$. Since $k<n$, we can take $B:=n=\max \{k,|-n|,|-1|\}$. Using the inequality (3.11) and Theorem 2.1 , we get

$$
\begin{aligned}
\frac{3}{\alpha^{n}} & >\left|\Lambda_{4}\right| \\
& >\exp \left(-1.4 \cdot 30^{6} \cdot 3^{4.5} \cdot 4^{2}(1+\log 4)(1+\log n) \cdot 3.6 \cdot 1 \cdot 4(1.26+m \log \alpha)\right),
\end{aligned}
$$

or

$$
n \log \alpha-\log 3<1.9681 \times 10^{12} \cdot(1+\log n) \cdot(5.04+4 m \log \alpha) .
$$

Inserting the inequality (3.10) into the last inequality, a computer search with Mathematica gives us that $n<6.26482 \cdot 10^{31}$. Now we reduce this bound to a size that can be easily dealt. In order to do this, we use Lemma 2.2 again. Let $z_{3}=k \log \lambda-(n+m) \log \alpha+\log (5 / 4 \sqrt{2})$. Then from the inequality (3.9), it follows that

$$
\left|1-e^{z_{3}}\right|<\frac{1}{\alpha^{2 m-1}}
$$

If $z_{3}>0$, then

$$
\left|z_{3}\right|=z_{3}<e^{z_{3}}-1=\left|1-e^{z_{3}}\right|<\frac{1}{\alpha^{2 m-1}}
$$

If $z_{3}<0$, then

$$
1-e^{z_{3}}=\left|1-e^{z_{3}}\right|<\frac{1}{\alpha^{2 m-1}}<\frac{2}{3}
$$


Thus $e^{-z_{3}}<3$, which yields to

$$
\left|z_{3}\right|<e^{\left|z_{3}\right|}-1=e^{\left|z_{3}\right|}\left|1-e^{z_{3}}\right|<\frac{3}{\alpha^{2 m-1}} .
$$

Therefore, it holds that

$$
\left|z_{3}\right|<\frac{3}{\alpha^{2 m-1}}
$$

Then

$$
|k \log \lambda-(n+m) \log \alpha+\log (5 / 4 \sqrt{2})|<\frac{3}{\alpha^{2 m-1}} .
$$

Dividing both sides of this inequality by $\log \alpha$, we get

$$
0<\left|k \frac{\log \lambda}{\log \alpha}-(n+m)+\frac{\log (5 / 4 \sqrt{2})}{\log \alpha}\right|<10.08 \cdot \alpha^{-2 m} .
$$

Now, we apply Lemma 2.2. Take $\gamma=: \log \lambda / \log \alpha, \mu:=\log (5 / 4 \sqrt{2}) / \log \alpha, A:=$ $10.08, B:=\alpha, w=2 m$ and $M=6.26482 \cdot 10^{31}$. We see that $q_{62}$, the denominator of the $62^{\text {th }}$ convergent of $\gamma$ exceeds $6 M$. Note that $M=6.26482 \cdot 10^{31}=n>k$. In this case, a quick computation with Mathematica gives us the inequality

$$
0<\epsilon=\left\|\mu q_{62}\right\|-M\left\|\gamma q_{62}\right\| \leq 0.39276 .
$$

Thus, with the help of Mathematica, we can say that the inequality (3.13) has no solution for

$$
2 m=w \geq \frac{\log \left(A q_{62} / \epsilon\right)}{\log B} \geq 163.277 .
$$

Therefore $m \leq 81$. Substituting this value of $m$ into (3.12), we get $n<2.70817$. $10^{27}$. Now, let

$$
z_{4}:=k \log \lambda-n \log \alpha+\log \left(\sqrt{5} / 4 \sqrt{2} F_{m}\right) .
$$

Then, from (3.11), we can write

$$
\left|1-e^{z_{4}}\right|<\frac{3}{\alpha^{n}}<\frac{1}{2}
$$

If $z_{4}>0$, then

$$
\left|z_{4}\right|=z_{4}<e^{z_{4}}-1=\left|1-e^{z_{4}}\right|<\frac{3}{\alpha^{n}} .
$$

If $z_{4}<0$, then $1-e^{z_{4}}=\left|1-e^{z_{4}}\right|<1 / 2$ and we get $e^{\left|z_{4}\right|}<2$. Thus,

$$
\left|z_{4}\right|<e^{\left|z_{4}\right|}-1=e^{\left|z_{4}\right|}\left|1-e^{z_{4}}\right|<\frac{6}{\alpha^{n}} .
$$

In both cases, it holds that $\left|z_{4}\right|<6 / \alpha^{n}$. That is,

$$
\left|k \log \lambda-n \log \alpha+\log \left(\sqrt{5} / 4 \sqrt{2} F_{m}\right)\right|<\frac{6}{\alpha^{n}} .
$$


Dividing both sides of this inequality by $\log \alpha$, we get

$$
0<\left|k \frac{\log \lambda}{\log \alpha}-n+\frac{\log \left(\sqrt{5} / 4 \sqrt{2} F_{m}\right)}{\log \alpha}\right|<12.46 \cdot \alpha^{-n} .
$$

Now, we apply Lemma 2.2. Let $\gamma:=\log \lambda / \log \alpha, \mu=\log \left(\sqrt{5} / 4 \sqrt{2} F_{m}\right) / \log \alpha, A:=$ $12.46, B:=k, w:=n$ and $M:=2.70817 \cdot 10^{17}$. It is seen that $q_{39}$, the denominator of the $39^{\text {th }}$ convergent of $\gamma$ exceeds $6 M$. Moreover, $M=n>k$. In this case, a quick computation with Mathematica gives us the inequality

$$
0<\epsilon=\left\|\mu q_{39}\right\|-M\left\|\gamma q_{39}\right\| \leq 0.493976
$$

for all $m \in[1,81]$. Thus, with the help of Mathematica, we can say that the inequality (3.14) has no solution for

$$
n=w \geq \frac{\log \left(A q_{39} / \epsilon\right)}{\log B} \geq \frac{\log \left(A q_{39} / 0.493976\right)}{\log B} \geq 105.224 .
$$

Therefore, $n \leq 105$. But this contradicts the assumption that $n>107$. This completes the proof.

\section{References}

[1] A. Baker, H. Davenport: The equations $3 x^{2}-2=y^{2}$ and $8 x^{2}-7=z^{2}$, Quart. J. Math. Oxford Ser. (2) 20.1 (1969), pp. 129-137, DOI: 10.1093/qmath/20.1.129.

[2] Y. Bugeaud, M. Mignotte, S. Siksek: Classical and modular approaches to exponential Diophantine equations I. Fibonacci and Lucas perfect powers, Ann. of Math. 163.3 (2006), pp. 969-1018, DOI: 10.4007/annals.2006.163.969.

[3] M. Ddamulira, F. Luca, M. Rakotomalala: Fibonacci Numbers which are products of two Pell numbers, The Fibonacci Quarterly 54.1 (2016), pp. 11-18.

[4] P. K. Dey, S. S. Rout: Diophantine equations concerning balancing and Lucas balancing numbers, Arch. Math. 108.1 (2017), pp. 29-43, DOI: 10.1007/s00013-016-0994-z.

[5] A. Dujella, A. Pethö: A generalization of a theorem of Baker and Davenport, Quart. J. Math. Oxford Ser. (2) 49.3 (1998), pp. 291-306, DOI: 10.1093/qmathj/49.3.291.

[6] R. Keskin, O. Karaatli: Some New Properties of Balancing Numbers and SquareTriangular Numbers, Journal of Integer Sequences (2012), Article 12.1.4, 13 pp.

[7] T. Koshy: Fibonacci and Lucas Numbers With Applications, New York: Wiley-Interscience Pub., 2001, DOI: 10.1002/9781118033067.

[8] E. M. Matveev: An Explicit lower bound for a homogeneous rational linear form in the logarithms of algebraic numbers II, (Russian), Izv. Ross. Akad. Nauk Ser. Mat. 64.6 (2000), pp. 125-180, Translation in Izv. Math. 64.6 (2000) pp. 1217-1269, DOI: 10.1070/ im2000v064n06abeh000314.

[9] G. K. PANDA: Some fascinating properties of balancing numbers, Proc. Eleventh Internat. Conference on Fibonacci Numbers and Their Applications, Cong. Numerantium 194 (2009), pp. 185-189.

[10] G. K. PAnda, P. K. RAY: Cobalancing numbers and cobalancers, Int. J. Math. Math. Sci. 8 (2005), pp. 1189-1200, DOI: 10.1155/ijmms.2005.1189. 
[11] S. S. Rout: Some Generalizations and Properties of Balancing Numbers, Ph.D. Thesis, NIT Rourkela, 2015.

[12] T. SzaKÁcs: Multiplying balancing numbers, Acta Univ Sapientiae Mat. 3.1 (2011), pp. 9096. 